\title{
Accuracy of Magnetic Resonance Imaging and Computed Tomography Arthrography in Diagnosing Acetabular Labral Tears and Chondral Lesions
}

\author{
Guen Young Lee, MD, Sujin Kim, MD, Suk-Ho Baek, MD*, Eui-Chan Jang, MD*, Yong-Chan Ha, MD*
}

Departments of Radiology and * Orthopaedic Surgery, Chung-Ang University College of Medicine, Seoul, Korea

Background: We investigated sensitivity, specificity, and accuracy of magnetic resonance imaging (MRI) and computed tomography arthrography (CTA), on the basis of arthroscopic findings, to diagnose acetabular labral tears and chondral lesions.

Methods: We retrospectively reviewed the results of MRI and subsequent CTA in 36 hips that underwent arthroscopic surgery (33 patients; 17 males [17 hips] and 16 females [19 hips]; average age, 35 years). All patients had positive impingement test results and groin pain. We analyzed sensitivity, specificity, and accuracy of MRI and CTA by comparing with the arthroscopic findings. Interobserver agreement and intraobserver reproducibility of the presence of tears and cartilage lesions on MRI and CTA were calculated using Kappa coefficients.

Results: The sensitivity, specificity, and accuracy of MRI for detection of acetabular labral tears by two observers were $60 \%, 80 \%$, and $64 \%$, respectively, and $65 \%, 70 \%$, and $69 \%$, respectively. The sensitivity, specificity, and accuracy of CTA for detection of labral tears by both observers were $85 \%, 90 \%$, and $86 \%$, respectively, and $92 \%, 80 \%$, and $89 \%$, respectively. However, the sensitivity and specificity of MRI for detection of acetabular chondral lesions by both observers were $36 \%$ and $84 \%$, respectively, and $46 \%$ and $88 \%$, respectively. The sensitivity and specificity of CTA for detecting acetabular cartilage lesions by both observers were $46 \%$ and $72 \%$, respectively, and $64 \%$ and $72 \%$, respectively. Intraobserver reproducibility for detection of labral tears and chondral lesions by using MRI was substantial ( $\kappa=0.756$ and $\kappa=0.693$, respectively). Interobserver reliability for detection of labral tears and chondral lesions by using MRI was substantial ( $\kappa=0.700$ and $\kappa=0.875$, respectively). Intraobserver reproducibility for detection of labral tears and chondral lesions by using CTA was substantial ( $\kappa=0.832$ and $\kappa=0.774$, respectively). Interobserver reliability for detection of labral tears and chondral lesions by using CTA was high ( $\kappa=0.886$ and $\kappa=0.596$, respectively).

Conclusions: This study demonstrated that the accuracy of MRI to detect an acetabular labral tear and a chondral lesion of the hip joint was not sufficient. CTA was reliable in the diagnosis of acetabular labral tears. However, both CTA and MRI were also of limited value to detect chondral lesions.

Keywords: Hip, Magnetic resonance imaging, Computed tomography arthrography, Acetabular labrum, Chondral lesion

Received July 24, 2018; Accepted October 28, 2018

Correspondence to: Yong-Chan Ha, MD

Department of Orthopaedic Surgery, Chung-Ang University College of

Medicine, 102 Heukseok-ro, Dongjak-gu, Seoul 06973, Korea

Tel: +82-2-6299-1577, Fax: +82-2-822-1710

E-mail: hayongch@naver.com

Copyright (c) 2019 by The Korean Orthopaedic Association

This is an Open Access article distributed under the terms of the Creative Commons Attribution Non-Commercial License (http://creativecommons.org/licenses/by-nc/4.0) which permits unrestricted non-commercial use, distribution, and reproduction in any medium, provided the original work is properly cited. 
Acetabular labral and cartilage lesions in femoroacetabular impingement are considered causal factors of earlyonset osteoarthritis. ${ }^{1)}$ Recent improvements in the quality of computed tomography (CT) arthrography (CTA) with multi-detector CT (MDCT) with submillimeter spatial resolution has increased the use of CTA for evaluating the cartilage, ligament, and labral lesions in various joints. ${ }^{2-4)}$ Although several recent studies have documented the use of magnetic resonance imaging (MRI) for diagnosing acetabular labral and chondral lesions, validation data have been inconsistent. ${ }^{5-7)}$ In addition, there has been only one study examining the diagnostic sensitivity and specificity of MRI and CTA in dysplastic hip joints. ${ }^{4)}$ The purpose of this study was to investigate the sensitivity, specificity, and accuracy of MRI and CTA for diagnosing acetabular labral tears using the Lage classification system ${ }^{8)}$ and chondral lesions compared with arthroscopic findings.

\section{METHODS}

The design and protocol of this retrospective study were approved by the Institutional Review Board of ChungAng University Hospital (IRB No. C2014078(1274)) and informed consent was waived.

\section{Patient Selection}

A retrospective review of 33 patients (36 hips) who underwent MRI and direct CTA of the hip and arthroscopic surgery from January 2010 to December 2014 was conducted. The inclusion criteria were patients who had groin pain and positive results on the impingement test or the flexion, abduction, and external rotation test and patients who underwent arthroscopic surgery within 2 months after CTA. The 33 patients ( 36 hips) comprised 17 males (17 hips, 47\%) and 16 females (19 hips, 53\%). The mean age of the patients at the time of diagnosis was 35 years (range, 20 to 61 years). None of the patients had previously undergone surgery on the ipsilateral hip joint. The demographic features of the patients including age, sex, and primary diagnosis are presented in Table 1.

\section{MRI Protocol}

MRI was performed on a 1.5-T (20 patients; Philips Medical Systems, Best, the Netherlands; Siemens Medical Solutions, Erlangen, Germany; GE Healthcare, Milwaukee, WI, USA) or 3.0-T (13 patients; Philips Medical Systems) scanner. The MRI protocol included three imaging planes except one patient. Axial, sagittal, and coronal images were obtained with T1-weighted image (T1WI) or T2WI or proton density weighted image (PDWI) sequences. T2WI and PDWI were with or without fat saturation. The ranges of repetition time and echo time were variable (4041,041/8-20 ms for T1WI, 2,120-9,063/23-118 ms for T2WI fat spin echo and 1,700-3,500/10-40 ms for PDWI). Other imaging parameters were as follows: matrix, $224 \times$ $224-512 \times 512$; field of view, $22-43 \mathrm{~cm}$; slice thickness, $3-8$ $\mathrm{mm}$; and number of signals averaged, 1 or 2 .

\section{CTA Protocol}

Under ultrasound guidance, a 21-G spinal needle was placed in the hip joint by an anterior approach using a standardized aseptic technique. A total of $10-12 \mathrm{~mL}$ mixture of $10 \mathrm{~mL}$ iopamidol and a solution of $5 \mathrm{~mL}$ of normal saline and $0.3 \mathrm{~mL} 2 \%$ lidocaine was injected into the hip joint. CT scans were obtained with a 16-slice MDCT or 256-slice MDCT with standard acquisition protocols as follows: for 16-channel MDCT, pitch, 0.938; current, 10-40 mAs; voltage, $120 \mathrm{kVp}$; collimation, $10 \mathrm{~mm}$; field of view at acquisition, $15-20 \mathrm{~cm}$; slice thickness, $2.5 \mathrm{~mm}$ without section overlapping; and matrix size, $512 \times 512$; for 256-channel MDCT, pitch, 0.579; current, 200-300 $\mathrm{mAs}$; voltage, $120 \mathrm{kVp}$; collimation, $40 \mathrm{~mm}$; field of view at acquisition, $15-20 \mathrm{~cm}$; slice thickness, $2.5 \mathrm{~mm}$ without section overlapping; and matrix size, $512 \times 512$. Coronal, sagittal, and oblique axial images (parallel to the femoral neck axis) were routinely reconstructed using an EBW 4.5 workstation after axial scanning.

\section{Image Analysis}

Two experienced musculoskeletal radiologists (GYL and SK) independently reviewed the MRI and CTA scans in

\begin{tabular}{|c|c|}
\hline Variable & Value \\
\hline No. of patients & 33 (36 Hips) \\
\hline Male:female & 17 (17 Hips, 47):16 (19 Hips, 53) \\
\hline Age (yr) & $35 \pm 13.2(20-61)$ \\
\hline \multicolumn{2}{|l|}{ Primary diagnosis (hip) } \\
\hline Femoroacetabular impingement & $20(55.5)$ \\
\hline Pincer type & 8 \\
\hline Cam type & 8 \\
\hline Combined type & 4 \\
\hline Labral tear & $11(30.5)$ \\
\hline Snapping hip & $5(14)$ \\
\hline
\end{tabular}

Values are presented as number (\%) or mean \pm standard deviation (range). 
Lee et al. Diagnostic Accuracy of MRI and CTA

Clinics in Orthopedic Surgery • Vol. 11, No. 1, 2019•www.ecios.org

random order. After two radiologists came to consensus during review sessions, each examiner was blinded to other measurements and arthroscopic findings. The observers repeated the measurements in separate sessions.

\section{MRI Interpretation}

All images were evaluated for the presence of a labral tear and cartilage lesion. Labral abnormalities were classified into three types using an established classification system $^{2,9,10)}$ as follows: type 1 (radial flap tear), a tear with a discrete linear high signal intensity extending either partially or all the way through the labral substance, creating a flap; type 2 (radial fibrillated tear), irregular margins of the labrum without a discrete tear; and type 3 (longitudinal peripheral or base tear), a tear with a linear high signal intensity extending partially or completely between the labral base and acetabulum labral detachment. The cartilage lesion was graded using a 3-point scale: 1, complete visualization-sharp edges; 2 , blurred edges fissuringpartial defects; and 3, exposed bone. ${ }^{11)}$

\section{CTA Image Interpretation}

An acetabular labral abnormality was diagnosed when the inflow of contrast medium was observed to be continuous with the joint space at the base of the acetabular labrum. ${ }^{12)}$ Labral blunting, displacement, or intrasubstance contrast material were also considered a tear. Linear contrast material at the acetabular-labral junction also indicated a tear if the margins were irregular or a sublabral sulcus if the margins were smooth.

Each labral tear or detachment on CTA was then classified by Blankenbaker modification of the Lage arthroscopic classification. ${ }^{2,8,9)}$ Tears were classified as arthrographic Lage type 1, 2, 3, or 4. A type 1 tear (radial flap tear) was diagnosed on CTA if a discrete contrast cleft was seen extending either partially or all the way through the labral substance creating a flap. A type 2 tear (radial fibrillated) was diagnosed if there was irregularity of the labral outline, but no discrete cleft within the labrum. A type 3 tear (longitudinal peripheral) was diagnosed when there was contrast extending through the labrum either very near or at the junction between the labrum and the acetabulum. A type 4 unstable tear was diagnosed if the labrum had a thickened and distorted appearance. These criteria have their limitations because a torn labrum often becomes thickened and distorted when it is unstable, whereas not all unstable tears are thickened. Such tears were classified into the most closely appropriate type.

\section{Arthroscopy}

Thirty-six hips underwent arthroscopic surgery within 2 months after CTA. A single arthroscopic surgeon $(\mathrm{YCH})$ performed all operations using a technique described by Byrd. ${ }^{13,14)}$ A tear was identified by passing a hook between the labrum and the subchondral bone. Two experienced orthopedic surgeons who were not involved in the preoperative image evaluations (SHB and ECJ) took part in each operation and arrived at a consensus on the interpretation of the arthroscopic findings during and after surgery using movie clips and photos.

\section{Statistical Analyses}

Both orthopedic surgeons who were not involved in the image evaluation assessed the arthroscopic findings and compared with the MRI findings and CTA findings to evaluate sensitivity, specificity, and accuracy of MRI and CTA. Inter- and intraobserver reliabilities for the measured parameters including labral tears and chondral lesions in 36 hips were assessed using Kappa coefficients. These were interpreted as follows: $0.00-0.20$, poor; $0.21-$ 0.40 , fair; $0.41-0.60$, moderate; $0.61-0.80$, substantial; and $>0.80$, almost perfect agreement. ${ }^{15)}$ The Kappa coefficient method was used for statistical analysis of comparison of MRI classification and modified CT classification of the Blankenbaker with the arthroscopic findings of the Lage arthroscopic classification.

Chi-square or Fisher exact test was used to compare

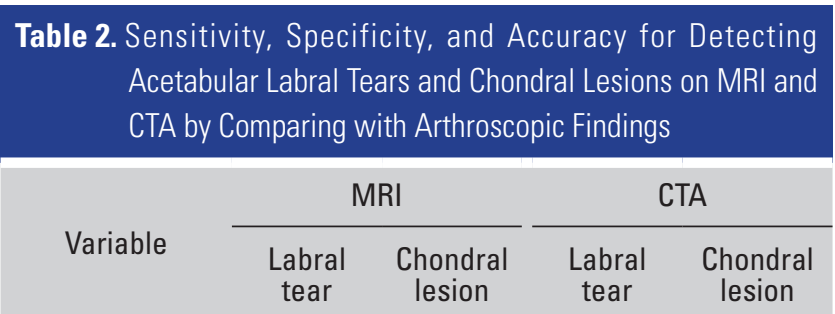

Observer 1

\begin{tabular}{|lllll}
\hline Sensitivity (\%) & 60 & 36 & 85 & 46 \\
\hline Specificity (\%) & 80 & 84 & 90 & 72 \\
\hline Accuracy (\%) & 64 & 69 & 86 & 64 \\
\hline Observer 2 & & & & \\
\hline Sensitivity (\%) & 65 & 46 & 92 & 64 \\
\hline Specificity (\%) & 70 & 88 & 80 & 72 \\
\hline Accuracy (\%) & 69 & 75 & 89 & 69 \\
\hline
\end{tabular}

MRI: magnetic resonance imaging, CTA: computed tomography arthrography. 
arthroscopic findings with the acetabular labral lesions and chondral lesions observed on CTA and MRI. We used SAS ver. 6.2 (SAS Institute, Cary, NC, USA). Statistical significance was defined as a $p$-value $<0.05$.

\section{RESULTS}

Arthroscopy of the 36 hips revealed an acetabular labral tear in 26 hips and no abnormality in 10 hips. MRI and CTA of the 36 hips revealed an acetabular labral tear in 17 hips and 23 hips, respectively, for observer $1(p=0.032)$ and in 21 hips and 26 hips, respectively, for observer $2(p<$ 0.001). The sensitivity, specificity, and accuracy of MRI for detection of labral tears were $60 \%, 80 \%$, and $64 \%$, respectively, for observer 1 and 65\%,70\%, and 69\%, respectively, for observer 2 (Table 2). The sensitivity, specificity, and accuracy of CTA for detection of labral tears were $85 \%, 90 \%$, and $86 \%$, respectively, for observer 1 and $92 \%, 80 \%$, and 89\%, respectively, for observer 2 (Table 2 and Fig. 1).
Arthroscopy of the 36 hips revealed chondral lesions in 11 hips, including a carpet lesion without cartilage defect in two hips and cartilage defects in seven hips. On MRI and CTA of 36 hips, a chondral lesion was found in eight hips and 12 hips, respectively, for observer 1 ( $p$ $=0.369)$ and in eight hips and 14 hips, respectively, for observer $2(p=0.050)$. The sensitivity, specificity, and accuracy of MRI for detection of chondral lesions were $36 \%, 84 \%$, and $69 \%$, respectively, for observer 1 and $46 \%$, $88 \%$, and $75 \%$, respectively, for observer 2 (Table 2). The sensitivity, specificity, and accuracy of CTA for detection of chondral lesions were $46 \%, 72 \%$, and $64 \%$, respectively, for observer 1 and $64 \%, 72 \%$, and 69\%, respectively, for observer 2 (Table 2 and Fig. 2).

According to the Lage morphologic classification, arthroscopic examination revealed longitudinal peripheral tears in 18 hips, four radial fibrillated tears, two radial flaps, and two unstable tears. The MRI findings of observer 1 include 13 hips with longitudinal peripheral tears.
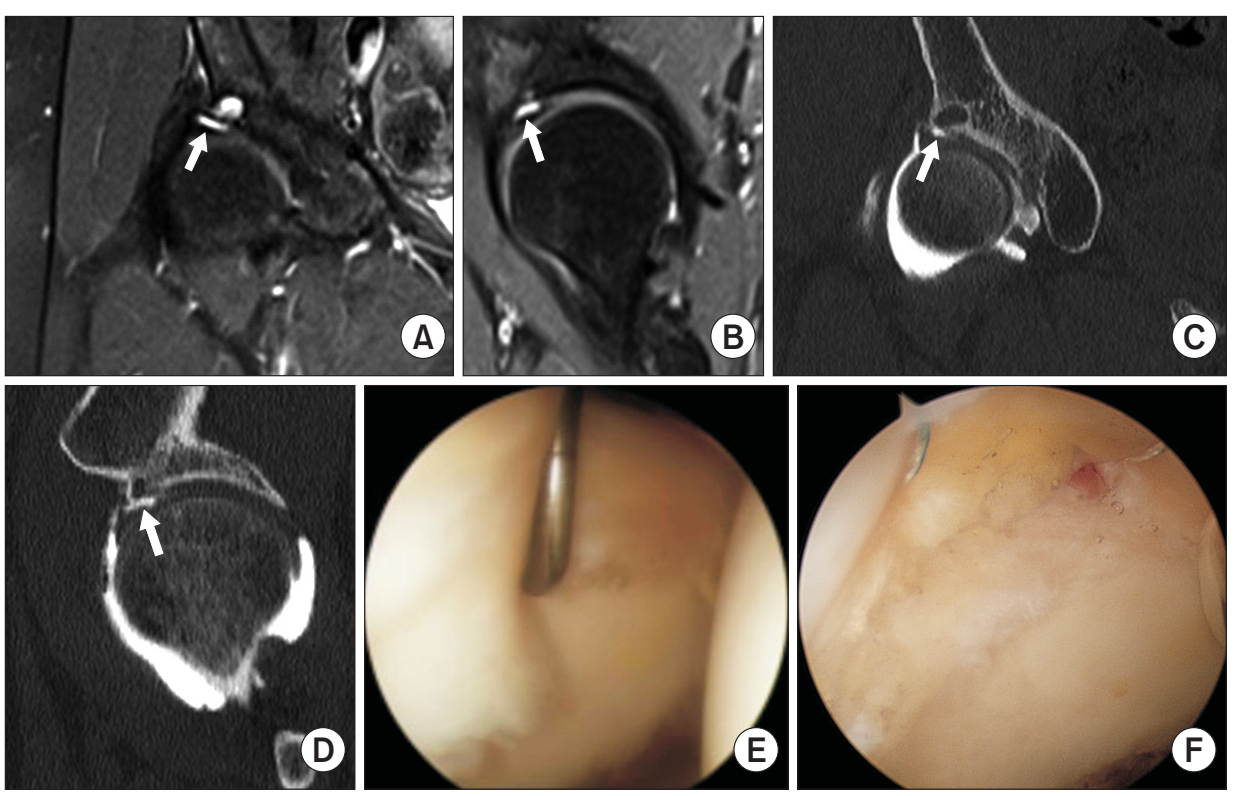

Fig. 1. Proton density magnetic resonance imaging (MRI): the coronal $(\mathrm{A})$ and sagittal (B) views showed a cartilage defect (arrows) on the right acetabulum. The defective cartilage lesion was also seen in computed tomography arthrography (CTA): coronal (C) and sagittal (D) views (arrows). Adjacent subchondral cyst of the acetabulum just above this chondral defect was observed in MRI and CTA. (E) Labral tear. (F) This lesion could be confirmed in arthroscopic examination after labral repair.
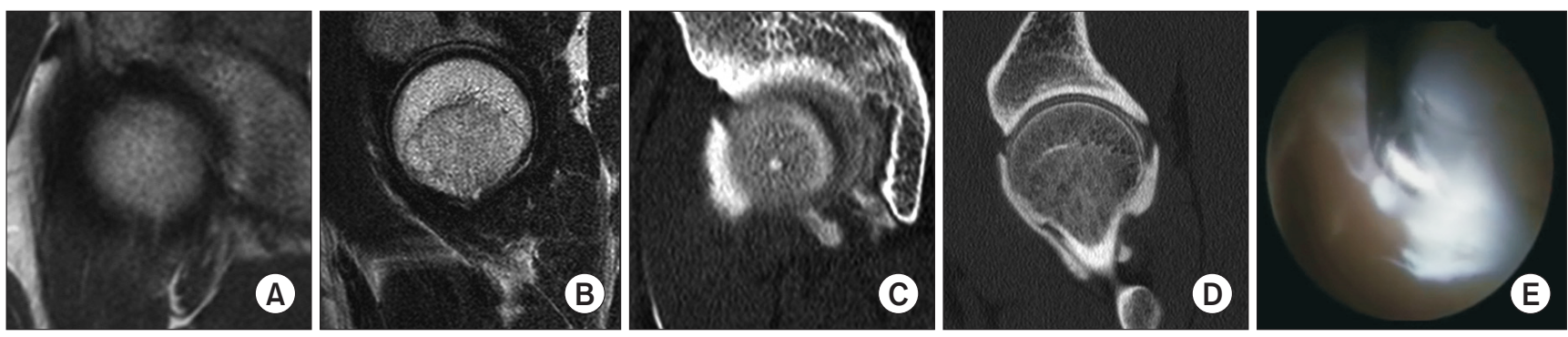

Fig. 2. On T2-weighted magnetic resonance imaging coronal $(A)$ and sagittal $(B)$ views, a definite labral tear was not identified, whereas the lesion was observed in computed tomography coronal (C) and sagittal (D) views. (E) This lesion was confirmed in arthroscopic examination. 


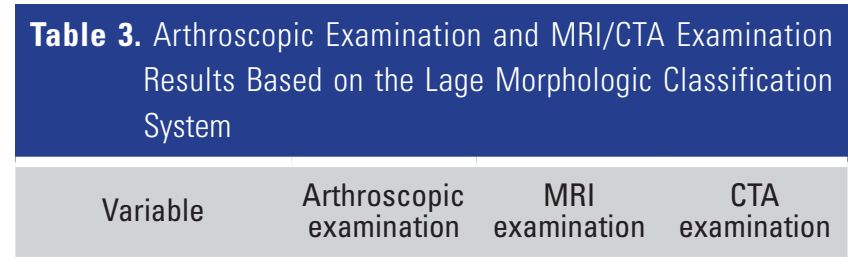

\begin{tabular}{lccc}
\hline Observer 1 & & \\
\hline $\begin{array}{c}\text { Longitudinal } \\
\text { peripheral tear }\end{array}$ & 18 & 13 & 16 \\
\hline Radial fibrillation & 4 & 1 & 3 \\
\hline Radial flap & 2 & 1 & 1 \\
\hline Unstable & 2 & 2 & 3 \\
\hline Observer 2 & 18 & 15 & 15 \\
\hline Longitudinal \\
peripheral tear
\end{tabular}

MRI: magnetic resonance imaging, CTA: computed tomography arthrography.
The CTA findings of observer 1 include 16 hips with longitudinal peripheral tears, three radial fibrillated tears, one flap tear, and three unstable tears (Table 3). Compared to the morphologic classification based on arthroscopic findings, the accuracy of MRI was 53\% for observer 1 and $62 \%$ for observer 2; the accuracy of CTA was $65 \%$ for observer 1 and $72 \%$ for observer 2 .

Intraobserver reproducibility for MRI detection of labral tears and chondral lesions was substantial $(\kappa=0.756$ and $\mathrm{K}=0.693$, respectively). Interobserver reliability for MRI detection of labral tears and chondral lesions was substantial ( $\mathrm{\kappa}=0.700$ and $\kappa=0.875$, respectively). Intraobserver reproducibility for CTA detection of labral tears and chondral lesions was substantial $(\kappa=0.832$ and $\kappa=0.774$, respectively). Interobserver reliability for MRI detection of labral tears and chondral lesions was substantial $(\kappa=0.886$ and $\kappa=0.596$, respectively).

\section{DISCUSSION}

Many clinicians recommend MRI for evaluating hip pathologies due to the convenience of the examination and less invasiveness than magnetic resonance arthrography (MRA). ${ }^{5,11,16,17)}$ However, the diagnostic value of MRI for acetabular labral and chondral lesions is not consis-

\section{Table 4. Comparative Studies of MRI, MRA, and CTA}

\begin{tabular}{|c|c|c|c|c|c|c|}
\hline Study & $\begin{array}{l}\text { Subject patient } \\
\text { (hip) }\end{array}$ & $\begin{array}{l}\text { Mean age } \\
(y r)\end{array}$ & Comparison & Sensitivity (\%) & Specificity (\%) & Accuracy $(\%)$ \\
\hline Byrd and Jones ${ }^{5)}$ & $40(40)$ & NR & $\begin{array}{l}\text { MRA vs. MRI } \\
\text { Labral tear } \\
\text { Chondral lesion }\end{array}$ & $\begin{array}{l}66 \text { vs. } 25 \\
41 \text { vs. } 18\end{array}$ & $\begin{array}{l}75 \text { vs. } 67 \\
100 \text { vs. } 100\end{array}$ & $\begin{array}{l}N R \\
N R\end{array}$ \\
\hline Nishii et al. ${ }^{4)}$ & $18(20)$ & $12-49$ & $\begin{array}{l}\text { CTA vs. MRI } \\
\text { Chondral lesion1 } \\
\text { Chondral lesion2 }\end{array}$ & $\begin{array}{l}67 \text { vs. } 49 \\
70 \text { vs. } 47\end{array}$ & $\begin{array}{l}89 \text { vs. } 89 \\
79 \text { vs. } 71\end{array}$ & $\begin{array}{l}79 \text { vs. } 71 \\
87 \text { vs. } 80\end{array}$ \\
\hline Czerny et al. ${ }^{6)}$ & $56(57)$ & 39 & $\begin{array}{l}\text { MRA vs. MRI } \\
\text { Labral tear }\end{array}$ & 90 vs. 30 & NR & 91 vs. 36 \\
\hline Perdikakis et al. ${ }^{11)}$ & $10(14)$ & 43 & $\begin{array}{l}\text { MRA vs. CTA } \\
\text { Labral tear } \\
\text { Chondral lesion }\end{array}$ & $\begin{array}{r}100 \text { vs. } 15 \\
63 \text { vs. } 66\end{array}$ & $\begin{array}{l}50 \text { vs. } 13 \\
33 \text { vs. } 40\end{array}$ & $\begin{array}{l}90 \text { vs. } 14 \\
55 \text { vs. } 66\end{array}$ \\
\hline This study & $33(36)$ & 35 & $\begin{array}{l}\text { CTA vs. MRI } \\
\text { Labral tear } \\
\text { Chondral lesion }\end{array}$ & $\begin{array}{l}85 \text { vs. } 60 \\
46 \text { vs. } 36\end{array}$ & $\begin{array}{l}90 \text { vs. } 80 \\
72 \text { vs. } 84\end{array}$ & $\begin{array}{l}86 \text { vs. } 64 \\
64 \text { vs. } 69\end{array}$ \\
\hline
\end{tabular}

MRI: magnetic resonance imaging, MRA: magnetic resonance arthrography, CTA: computed tomography arthrography, NR: not reported, Chondral lesion 1: homogeneous or focal signal intensity without surface irregularity, Chondral lesion 2: contour defect. 

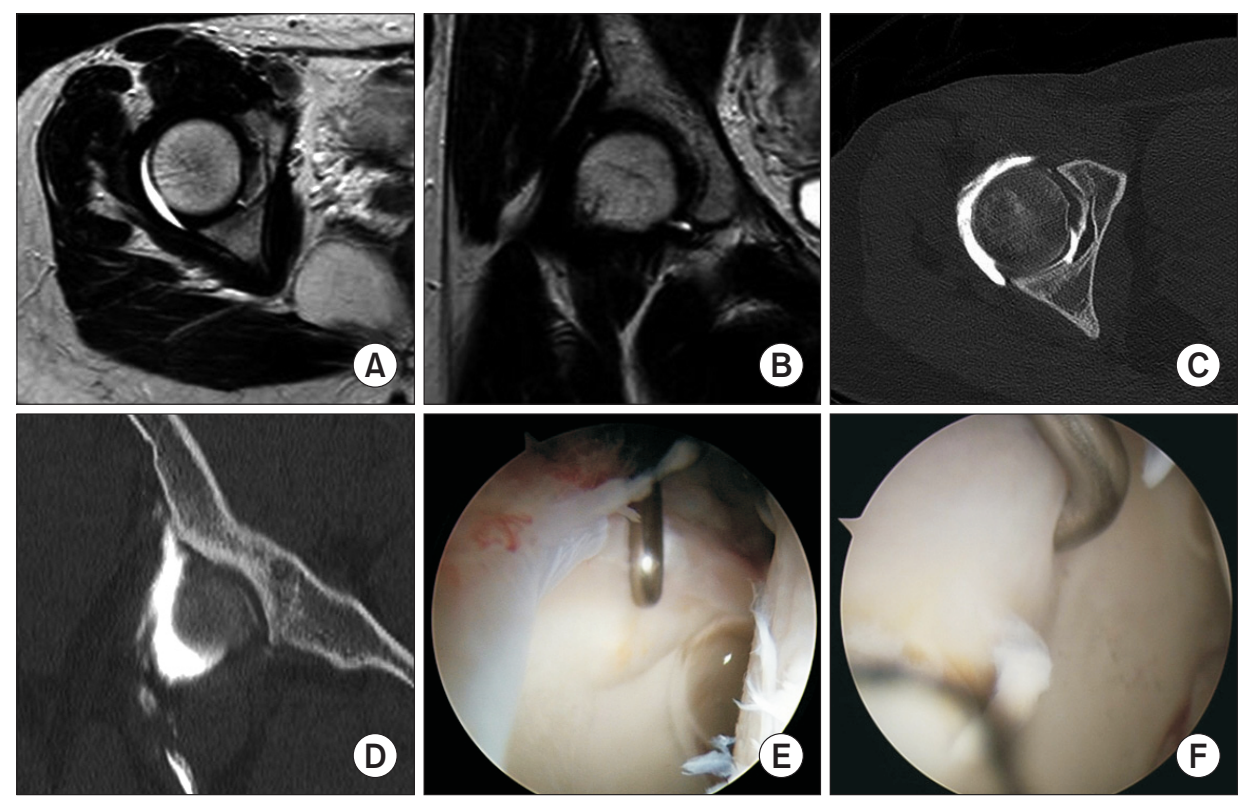

Fig. 3. There was no abnormal finding on the cartilage of the acetabulum in magnetic resonance imaging $(A, B)$ nor in computer tomography arthrography (C, D). But in arthroscopic examination, we could find a floating lesion of the cartilage (E), called "carpet lesion (F)."

tent. $^{5-7,17)}$ CTA has high merits for diagnosing acetabular labral lesions. ${ }^{2,3)}$ This is the first study that compared CTA with MRI. This comparative study has demonstrated higher sensitivity, specificity, and accuracy of CTA than MRI for detection of acetabular labral lesions. However, the diagnostic value of both CTA and MRI for detection of chondral lesions was unsatisfactory.

Sundberg et al. ${ }^{7)}$ reported that the diagnostic value of MRI for acetabular labral lesions was comparable to MRA. They performed a comparative study using $1.5-\mathrm{T}$ MRA and 3.0-T MRI for evaluating the acetabular labrum. They reported $100 \%$ sensitivity for MRI and $80 \%$ sensitivity for MRA. However, other studies have reported better sensitivity (range, 66\% to $100 \%$ ) and specificity (range, $50 \%$ to $100 \%$ ) of MRA than MRI for acetabular labral lesions. ${ }^{5,6,17)}$ In addition, there is no comparative study between CTA and MRI for acetabular labral lesions. Our results show that the diagnostic value of CTA is significantly better than MRI for acetabular labral tears.

Many studies have reported better outcomes of early surgical intervention in patients with early-stage osteoarthritis. ${ }^{18-20)}$ Therefore, detecting acetabular cartilage lesions at an early stage is important to establish an appropriate treatment plan. Although MRI and MRA remain the imaging techniques of choice to detect cartilage lesions, their diagnostic values to detect chondral lesions were limited (Table 4). In our study, the sensitivity and accuracy of CTA to detect chondral lesions were better than those of MRI. However, the sensitivity and accuracy of CTA for evaluating chondral lesions were $50 \%$ and $70 \%$, respectively (Table 4). We performed an evaluation to identify the reasons. Two hips showed normal cartilage conditions on both CTA and MRI. However, arthroscopic findings showed no cartilage defects or loss but carpet lesions (Fig. 3). ${ }^{21)}$ Nishii et al. ${ }^{4)}$ performed a comparative study using fat-suppressed three-dimensional fast spoiled gradientecho MRI and MDCTA. They evaluated cartilage lesions of 20 hips with acetabular dysplasia and reported that sensitivity and specificity of MRI and CTA for detecting any cartilage disorder without cartilage defects were $49 \%$ and $67 \%$, and $89 \%$ and $89 \%$, respectively, and sensitivity and specificity of MRI and CTA for detecting cartilage lesions with substance loss were $47 \%$ and $70 \%$, and $92 \%$ and $93 \%$, respectively. Therefore, conventional methods including MRA, MRI, and CTA may not be appropriate diagnostic methods if there were not cartilage defects.

Several limitations in this study should be noted. First, this study is a retrospective review of 36 hips that has a relatively low number of cases, and there is a possibility of selection bias. Second, all patients were symptomatic with mechanical hip pain and had a clinical suspicion of labral tears and chondral lesions. Therefore, the two radiologists may have presumed the presence of an acetabular labral tear or a chondral lesion during interpretation. Finally, MRI on nine patients (nine hips) performed at outside institutions may not have the same quality, negatively influencing the results.

This study demonstrated that the accuracy of MRI to evaluate an acetabular labral tear and chondral lesion of the hip joint was not sufficient. CTA was a reliable method in the diagnosis of acetabular labral tears. However, CTA and MRI are also of limited value to detect chondral lesions. 
Lee et al. Diagnostic Accuracy of MRI and CTA

Clinics in Orthopedic Surgery • Vol. 11, No. 1, 2019•www.ecios.org

\section{CONFLICT OF INTEREST}

No potential conflict of interest relevant to this article was reported.

\section{REFERENCES}

1. Harris WH. Etiology of osteoarthritis of the hip. Clin Orthop Relat Res. 1986;(213):20-33.

2. Jung JY, Kim GU, Lee HJ, Jang EC, Song IS, Ha YC. Diagnostic value of ultrasound and computed tomographic arthrography in diagnosing anterosuperior acetabular labral tears. Arthroscopy. 2013;29(11):1769-76.

3. Ha YC, Choi JA, Lee YK, et al. The diagnostic value of direct CT arthrography using MDCT in the evaluation of acetabular labral tear: with arthroscopic correlation. Skeletal Radiol. 2013;42(5):681-8.

4. Nishii T, Tanaka H, Nakanishi K, Sugano N, Miki H, Yoshikawa H. Fat-suppressed 3D spoiled gradient-echo MRI and MDCT arthrography of articular cartilage in patients with hip dysplasia. AJR Am J Roentgenol. 2005;185(2):37985 .

5. Byrd JW, Jones KS. Diagnostic accuracy of clinical assessment, magnetic resonance imaging, magnetic resonance arthrography, and intra-articular injection in hip arthroscopy patients. Am J Sports Med. 2004;32(7):1668-74.

6. Czerny C, Hofmann S, Neuhold A, et al. Lesions of the acetabular labrum: accuracy of MR imaging and MR arthrography in detection and staging. Radiology. 1996;200(1):22530 .

7. Sundberg TP, Toomayan GA, Major NM. Evaluation of the acetabular labrum at 3.0-T MR imaging compared with 1.5T MR arthrography: preliminary experience. Radiology. 2006;238(2):706-11.

8. Lage LA, Patel JV, Villar RN. The acetabular labral tear: an arthroscopic classification. Arthroscopy. 1996;12(3):269-72.

9. Blankenbaker DG, De Smet AA, Keene JS, Fine JP. Classification and localization of acetabular labral tears. Skeletal Radiol. 2007;36(5):391-7.

10. Jin W, Kim KI, Rhyu KH, et al. Sonographic evaluation of anterosuperior hip labral tears with magnetic resonance arthrographic and surgical correlation. J Ultrasound Med. 2012;31(3):439-47.
11. Perdikakis E, Karachalios T, Katonis P, Karantanas A. Comparison of MR-arthrography and MDCT-arthrography for detection of labral and articular cartilage hip pathology. Skeletal Radiol. 2011;40(11):1441-7.

12. Vande Berg BC, Lecouvet FE, Poilvache P, Maldague B, Malghem J. Spiral CT arthrography of the knee: technique and value in the assessment of internal derangement of the knee. Eur Radiol. 2002;12(7):1800-10.

13. Byrd JW. Avoiding the labrum in hip arthroscopy. Arthroscopy. 2000;16(7):770-3.

14. Byrd JW. Hip arthroscopy: the supine position. Clin Sports Med. 2001;20(4):703-31.

15. Landis JR, Koch GG. The measurement of observer agreement for categorical data. Biometrics. 1977;33(1):159-74.

16. Petersilge CA, Haque MA, Petersilge WJ, Lewin JS, Lieberman JM, Buly R. Acetabular labral tears: evaluation with MR arthrography. Radiology. 1996;200(1):231-5.

17. Toomayan GA, Holman WR, Major NM, Kozlowicz SM, Vail TP. Sensitivity of MR arthrography in the evaluation of acetabular labral tears. AJR Am J Roentgenol. 2006;186(2):449-53.

18. Cohen SB, Huang R, Ciccotti MG, Dodson CC, Parvizi J. Treatment of femoroacetabular impingement in athletes using a mini-direct anterior approach. Am J Sports Med. 2012;40(7):1620-7.

19. Laude F, Sariali E, Nogier A. Femoroacetabular impingement treatment using arthroscopy and anterior approach. Clin Orthop Relat Res. 2009;467(3):747-52.

20. Murphy S, Tannast M, Kim YJ, Buly R, Millis MB. Debridement of the adult hip for femoroacetabular impingement: indications and preliminary clinical results. Clin Orthop Relat Res. 2004;(429):178-81.

21. Pollard TC. A perspective on femoroacetabular impingement. Skeletal Radiol. 2011;40(7):815-8. 